\title{
Tactile-visual temporal ventriloquism: No effect of spatial disparity
}

\author{
MirJam KeEtels AND JeAn VROOMEN \\ Tilburg University, Tilburg, The Netherlands
}

\begin{abstract}
Participants made visual temporal order judgments (TOJs) about which of two lights appeared first while taskirrelevant vibrotactile stimuli delivered to the index finger were presented before the first and after the second light. Temporally misaligned tactile stimuli captured the onsets of the lights, thereby improving sensitivity on the visual TOJ task, indicative of tactile-visual (TV) temporal ventriloquism (Experiment 1). The size of this effect was comparable to auditory-visual (AV) temporal ventriloquism (Experiment 2). Spatial discordance between the TV stimuli, as in the AV case, did not harm the effect (Experiments 3 and 4). TV stimuli thus behaved like AV stimuli, demonstrating that spatial co-occurrence is not a necessary constraint for intersensory pairing to occur.
\end{abstract}

The question of how the different sensory modalities cooperate to form a coherent representation of the environment is the focus of much behavioral and neuroscientific research (Calvert, Spence, \& Stein, 2004). The most commonly held view among researchers in multisensory perception is what has been referred to as the assumption of unity, which states that as events from different modalities share more (amodal) properties, they will be more likely to originate from a common object or source (see, e.g., Bedford, 1989; Bertelson, 1999; Radeau, 1994; Stein \& Meredith, 1993; Welch, 1999; Welch \& Warren, 1980). Although there is dispute as to whether "higher cognitive factors" count as a common property (e.g., Bertelson, 1999), without doubt the two most important ones are considered to be commonality in space and time (e.g., Radeau, 1994). On this account, one would expect interactions between sense organs to be more likely to occur if their information arrives from approximately the same place at approximately the same time.

However, even though most studies confirm that, within certain limits, temporal synchrony is crucial (Bertelson, 1999; Ernst \& Banks, 2002), there is debate about the role of spatial correspondence (Murray et al., 2004; Teder-Sälejärvi, Di Russo, McDonald, \& Hillyard, 2005; Welch, DuttonHurt, \& Warren, 1986). A critical example of intersensory pairing in which spatial discordance has been shown not to play a role is temporal ventriloquism, which refers to the auditory-visual (AV) phenomenon of a sound in temporal proximity to a light attracting the temporal occurrence of that light (Aschersleben \& Bertelson, 2003; Bertelson \& Aschersleben, 2003; Fendrich \& Corballis, 2001; Morein-Zamir, Soto-Faraco, \& Kingstone, 2003; Scheier, Nijhawan, \& Shimojo, 1999; Stekelenburg \& Vroomen, 2005; Vroomen \& de Gelder, 2004). MoreinZamir et al. demonstrated temporal ventriloquism using a visual temporal order judgment (TOJ) task (see also Vroomen \& Keetels, 2006). Their participants were presented with pairs of lights at various stimulus onset asynchronies (SOAs) and were asked to judge which of the two appeared first. A task-irrelevant sound before the first light and another after the second improved sensitivity in the visual TOJ task (i.e., they lowered the just noticeable difference, or JND) as compared with synchronized sounds, thus demonstrating that the sounds captured the onsets of the lights and effectively pulled them farther apart in time. Crucially, Vroomen and Keetels demonstrated that this effect is unaffected by whether the sounds come from the same positions as the lights or from different ones, whether the sounds are static or move from left to right or right to left, or whether the sounds and lights come from the same or from opposite sides of fixation. These results therefore challenge the view that intersensory interactions in general require spatial correspondence between the components of a multisensory event.

In the present study, we further explored the role of spatial correspondence by examining a tactile-visual (TV) form of temporal ventriloquism evoked by vibrotactile stimuli. So far, temporal ventriloquism has only been studied with sounds that capture the onsets of lights (Aschersleben \& Bertelson, 2003; Bertelson \& Aschersleben, 2003; Fendrich \& Corballis, 2001; Morein-Zamir et al., 2003; Scheier et al., 1999; Stekelenburg \& Vroomen, 2005; Vroomen \& de Gelder, 2004). Sounds may be special, though, because they have high temporal precision, whereas their spatial attributes are only secondary (Kubovy \& Van Valkenburg, 2001). In comparison with sounds, vibrotactile stimuli may be less well-defined in time, whereas their spatial attributes are more important, if only because the initial coding of touch is somato- rather than tonotopic. Spatial discordance therefore may be more

J.Vroomen, j.vroomen@uvt.nl 
harmful for TV than for AV temporal ventriloquism. If, on the other hand, spatial discordance between tactile and visual stimuli does not affect temporal ventriloquism, it would lend further credit to the notion that space in general may not be a relevant dimension for intersensory pairing to occur.

In Experiment 1, we first explored whether tactile stimuli can actually capture the onset of a light, because this had not previously been demonstrated. Our participants judged which of two lights was presented first, while taskirrelevant vibrotactile stimuli were presented before the first and after the second light (see Figure 1A). Temporal ventriloquism should manifest itself by improving sensitivity (i.e., lowering JNDs) on the visual task if the interval between the tactile stimuli and the lights is $\sim 100 \mathrm{msec}$ rather than $\sim 0$ msec (Morein-Zamir et al., 2003; Scheier et al., 1999; Vroomen \& Keetels, 2006). A visual-only condition was also included at this stage, to check whether the tactile stimuli actually enhanced rather than interfered with performance. After confirming that temporal ventriloquism can indeed be evoked by TV stimuli, we compared it in the later experiments with $\mathrm{AV}$ temporal ventriloquism and explored whether spatial discordance between touch and light affects the size of the effect.

\section{EXPERIMENT 1}

\section{Method}

Participants. Fourteen students (3 left-handed, 11 right-handed) from Tilburg University were included in the analyses in exchange for course credits, after we removed 3 participants for whom no reliable psychometric functions could be determined. All of the participants reported normal hearing and touch, as well as normal or corrected-to-normal vision. They were tested individually and were unaware of the purpose of the experiment.

Stimuli. The participants sat at a table in a dimly lit soundproof booth. Head movements were precluded by a chinrest. A small red LED was placed at a central location on the table, $50 \mathrm{~cm}$ from the chinrest, and served as a fixation point. The visual stimuli were presented using two green LEDs, positioned on the table at $5^{\circ}$ below and $5^{\circ}$ above fixation (diameter of $0.5 \mathrm{~cm}$, luminance of $40 \mathrm{~cd} / \mathrm{m}^{2}$ ). See Figure 1B for a schematic view of the experimental setup. The tactile stimuli $(250 \mathrm{~Hz}, 10-\mathrm{msec}$ duration) were delivered by a Sanko Electric minivibrator (Type 1E120; Sanko Electric, Japan) with a diameter of $1.4 \mathrm{~cm}$. The vibrator was embedded in a foam cube placed directly under the fixation point. Half of the participants were instructed to place their left index finger into the foam cube, the other half their right index finger. The participants could see their arm and finger position. They made responses with their other hand by pressing one of two keys in a response box on the table (index finger for an "upper light first" response, thumb for "lower light first"). White noise was presented continuously throughout the experiment at $65 \mathrm{~dB}(\mathrm{~A})$ to mask any sound generated by the vibrotactile stimulator.

Design. Two within-subjects factors were used: tactile stimulation $(\sim 0$ - or $\sim 100$-msec interval between the tactile capture stimuli and the lights, or no tactile stimulation) and SOA between the two lights $(-75$, $-60,-45,-30,-15,+15,+30,+45,+60$, or +75 msec; negative values indicate that the lower light was presented first). The $\sim 0$ - and $\sim 100$-msec intervals represent the times between the onset of the first tactile stimulus and the first light and between the second light and the second tactile stimulus. These factors yielded 30 equiprobable conditions, each presented 20 times, for a total of 600 trials. Trials were randomly presented in four blocks of 150 trials each, with a short pause between blocks. Testing lasted approximately $1 \mathrm{~h}$.
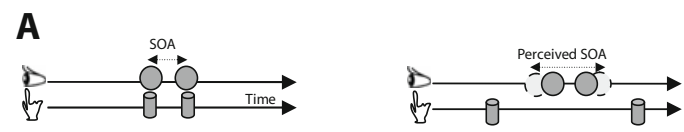

$\sim 0$-msec Tactile-Visual Interval $\sim 100-$ msec Tactile-Visual Interval
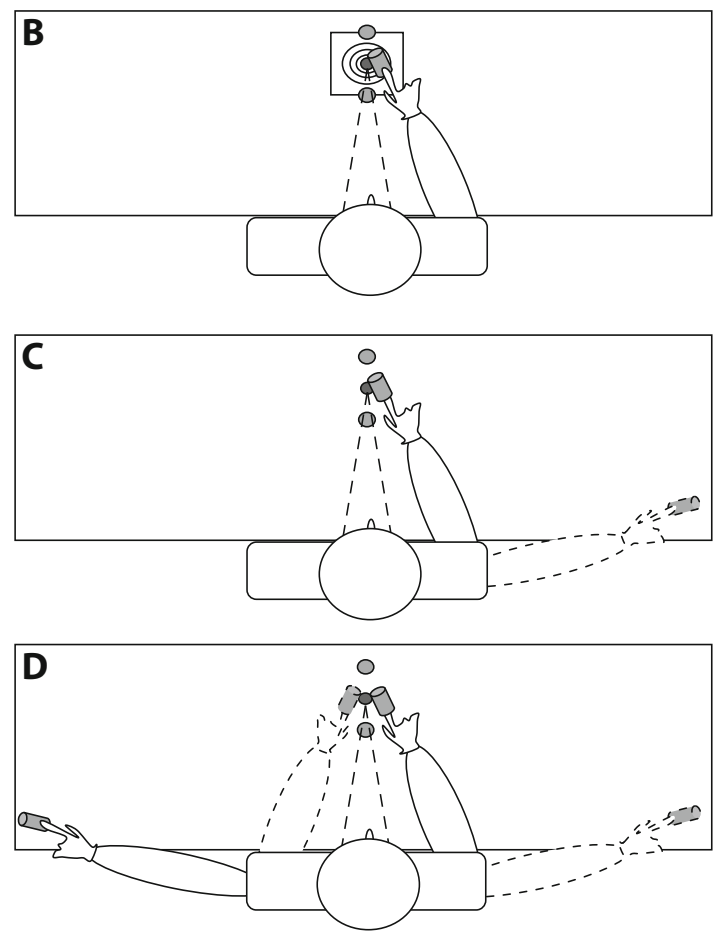

Target LED

g Vibrotactile Stimulator

- Fixation LED

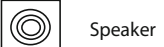

Figure 1. (A) A schematic illustration of a trial with vibrotactile capturing stimuli. Lights were presented at a particular SOA that ranged from -75 to $+75 \mathrm{msec}$, with negative values indicating that the lower light was presented first. Two vibrotactile stimuli were delivered on the index finger either simultaneously with the lights $(\sim 0-\mathrm{msec}$ interval) or $\sim 100 \mathrm{msec}$ before the first and $\sim 100$ msec after the second light ( $\sim 100-m s e c$ interval). Sensitivity in the visual temporal order judgment task was better in the $\sim 100$-msec than in the $\sim 0$-msec interval (i.e., temporal ventriloquism) because the two capturing stimuli "pulled" the lights farther apart, thereby increasing the apparent SOA between them. (B) Schematic setup of Experiments 1 and 2. Vibrotactile stimuli were delivered to the index finger. In Experiment 2, sounds were presented from the central location. (C) Schematic setup of Experiment 3. Vibrotactile stimuli were delivered to the index finger, which was positioned either close to the lights, in central position, or far from them, in a lateral position. The position of the touched finger varied between blocks. (D) Schematic setup of Experiment 4. Vibrotactile stimuli were delivered to either the left or the right index finger. One finger was positioned close to the lights, in central position, the other far away, in a lateral position. Which finger was touched (central or lateral) varied from trial to trial.

Procedure. The fixation light was illuminated at the beginning of the experiment. Participants were instructed to maintain fixation on the central red LED during testing. Trials consisted of the onset of the first green LED, followed after a particular SOA by 
the onset of the second green LED. Both LEDs remained lit until a response was made. Tactile stimuli were absent (visual-only condition), presented simultaneously with the lights ( $\sim 0$-msec interval), or presented $\sim 100 \mathrm{msec}$ before the first and after the second light ( 100-msec interval). The participants' task was to indicate whether the lower or upper LED was presented first by pressing the lower or the upper key on the response box. Responses were unspeeded, and participants were informed that the tactile stimuli should be ignored, since they did not predict in any way which light came first. The next trial started 2,000 msec after a response was detected.

To acquaint participants with the TOJ task, experimental blocks were preceded by a practice session. The practice block consisted of 24 trials in which all conditions were presented twice, in random order, at the largest SOAs ( \pm 75 and $\pm 60 \mathrm{msec}$ ). During practice, participants received verbal feedback ("Correct" or "Wrong") about whether they gave a correct or incorrect response.

\section{Results}

The trials in the practice session were excluded from analyses. The proportion of "upper-first" responses was calculated for each combination of tactile stimulation and SOA for each participant. The raw proportions were then converted into equivalent $z$ scores, assuming a cumulative normal distribution (Finney, 1964). For each condition, the best-fitting straight line was calculated over the 10 SOAs. The lines' slopes and intercepts were used to determine the JND (0.675/slope) and the point of subjective simultaneity (PSS $=50 \%$ "up" responses). The JND represents the smallest interval at which the temporal order of the two lights can be determined reliably, computed from the fitted line by calculating the difference between the SOAs at which $75 \%$ of the responses were "upper-first" and at which $25 \%$ of the responses were "upper-first," then dividing by two. The PSS represents the average interval by which the upper stimulus had to lead the lower one for them to be perceived as simultaneous (see Table 1 and Figure 2).

An ANOVA including tactile stimulation as a withinsubjects factor (visual-only, $\sim 0 \mathrm{msec}$, and $\sim 100 \mathrm{msec}$ ) was performed on the JNDs and PSSs. No effect emerged in the ANOVA on PSSs $(F<1)$, indicating that tactile stimuli did not shift the response distribution toward more "up" or "down" responses. More importantly, there was an effect of tactile stimulation on the JNDs $[F(2,26)=14.55$, $p<.001]$. A paired $t$ test showed, as expected, that JNDs were smaller (i.e., better sensitivity) with the $\sim 100$-msec than with the $\sim 0$-msec TV interval (30.9 vs. $38.5 \mathrm{msec}$ ), reflecting a 7.7-msec TV temporal ventriloquism effect (i.e., a $20 \%$ improvement) $[t(13)=2.75, p<.025]$. Moreover, simultaneous tactile stimuli enhanced rather than interfered with performance, because the visual-only condition had the largest JND $[t(13)=3.27, p<.01]$, greater than in the $\sim 0$-msec condition. The latter phenomenon has also been observed in AV temporal ventriloquism (see, e.g., Vroomen \& Keetels, 2006), in which it was shown that simultaneous sounds improve performance on a visual TOJ task, possibly because they sharpen the impulse response function of the flashes, reducing the overlap between them.

The results of Experiment 1 thus clearly demonstrate, for the first time, a TV temporal ventriloquist effect. Moreover, the comparison with the visual-only condition
Table 1

Mean Just Noticeable Differences (JNDs, in Milliseconds) and Standard Errors of the Means by Modality of the Capturing Stimuli, for Experiments 1 and 2

\begin{tabular}{|c|c|c|c|c|c|c|}
\hline \multirow[b]{3}{*}{ Interval } & \multicolumn{3}{|c|}{ Tactile } & \multicolumn{3}{|c|}{ Auditory } \\
\hline & \multicolumn{2}{|c|}{ JND } & \multirow[b]{2}{*}{ TVE } & \multicolumn{2}{|c|}{ JND } & \multirow[b]{2}{*}{ TVE } \\
\hline & $M$ & $\overline{S E}$ & & $M$ & $\overline{S E}$ & \\
\hline \multicolumn{7}{|c|}{ Experiment 1} \\
\hline$\sim 0 \mathrm{msec}$ & 38.5 & 4.4 & $7.7^{*}$ & & & \\
\hline$\sim 100 \mathrm{msec}$ & 30.9 & 2.5 & & & & \\
\hline Visual only & 48.7 & 5.1 & & & & \\
\hline \multicolumn{7}{|c|}{ Experiment 2} \\
\hline$\sim 0 \mathrm{msec}$ & 46.5 & 6.6 & $15.9^{*}$ & 38.5 & 5.9 & $13.4^{*}$ \\
\hline$\sim 100 \mathrm{msec}$ & 30.6 & 2.8 & & 25.1 & 1.7 & \\
\hline
\end{tabular}

Note - Tactile or auditory capturing stimuli were presented at $\sim 0$ - or $\sim 100$-msec intervals. The temporal ventriloquist effect (TVE) is the improvement in JND between the $\sim 0$ - and $\sim 100$-msec intervals. ${ }^{*} p<.05$.

excludes the possibility that the JND difference between the $\sim 0$ - and $\sim 100$-msec TV intervals was caused by a distracting effect from the co-occurring tactile stimuli. This allowed us to test further the resemblance between TV and AV temporal ventriloquism.

\section{EXPERIMENT 2}

In Experiment 2, we compared the strengths of AV and TV temporal ventriloquism. Unimodal studies have shown that the temporal resolution of the skin lies between those of hearing and vision (Kirman, 1973). According to the modality appropriateness hypothesis, one might then expect the TV temporal ventriloquist effect to be smaller than the AV effect (Welch \& Warren, 1980). However, in their classic study, Hirsh and Sherrick (1961) found that the JNDs in tactile performance were similar to those for hearing and vision, so no firm predictions can be made.

\section{Method}

Participants. Twelve students (all right-handed) from the same subject pool were tested, after 1 participant for whom no reliable psychometric function could be determined had been removed.

Stimuli and Design. These were as in Experiment 1, except for the following changes. The visual-only condition was replaced by an AV condition in which the auditory stimuli were presented via a hidden loudspeaker placed directly behind the central fixation LED. Three within-subjects factors were used: modality of the capturing stimuli (auditory or tactile), the interval between the capturing stimuli and the lights ( $\sim 0$ or $\sim 100 \mathrm{msec})$, and the SOA between the two lights (ranging from -75 to $+75 \mathrm{msec}$ in 10 steps of $15 \mathrm{msec}$ ). This yielded 40 equiprobable conditions, each presented 20 times (a total of 800 trials) in four blocks of 200 trials each. Practice consisted of 16 trials in which all conditions at the longest SOAs $( \pm 75$ and $\pm 60 \mathrm{msec}$ ) were presented randomly.

\section{Results and Discussion}

JNDs and PSSs were computed as before (see Table 1). A $2 \times 2$ ANOVA with the within-subjects factors modality of capturing stimulus (auditory or tactile) and interval ( $\sim 0$ or $\sim 100 \mathrm{msec}$ ) was performed on the JNDs and PSSs. In the ANOVA on PSSs, a main effect of modality of the capturing stimuli was found $[F(1,11)=34.03, p<.001]$, 

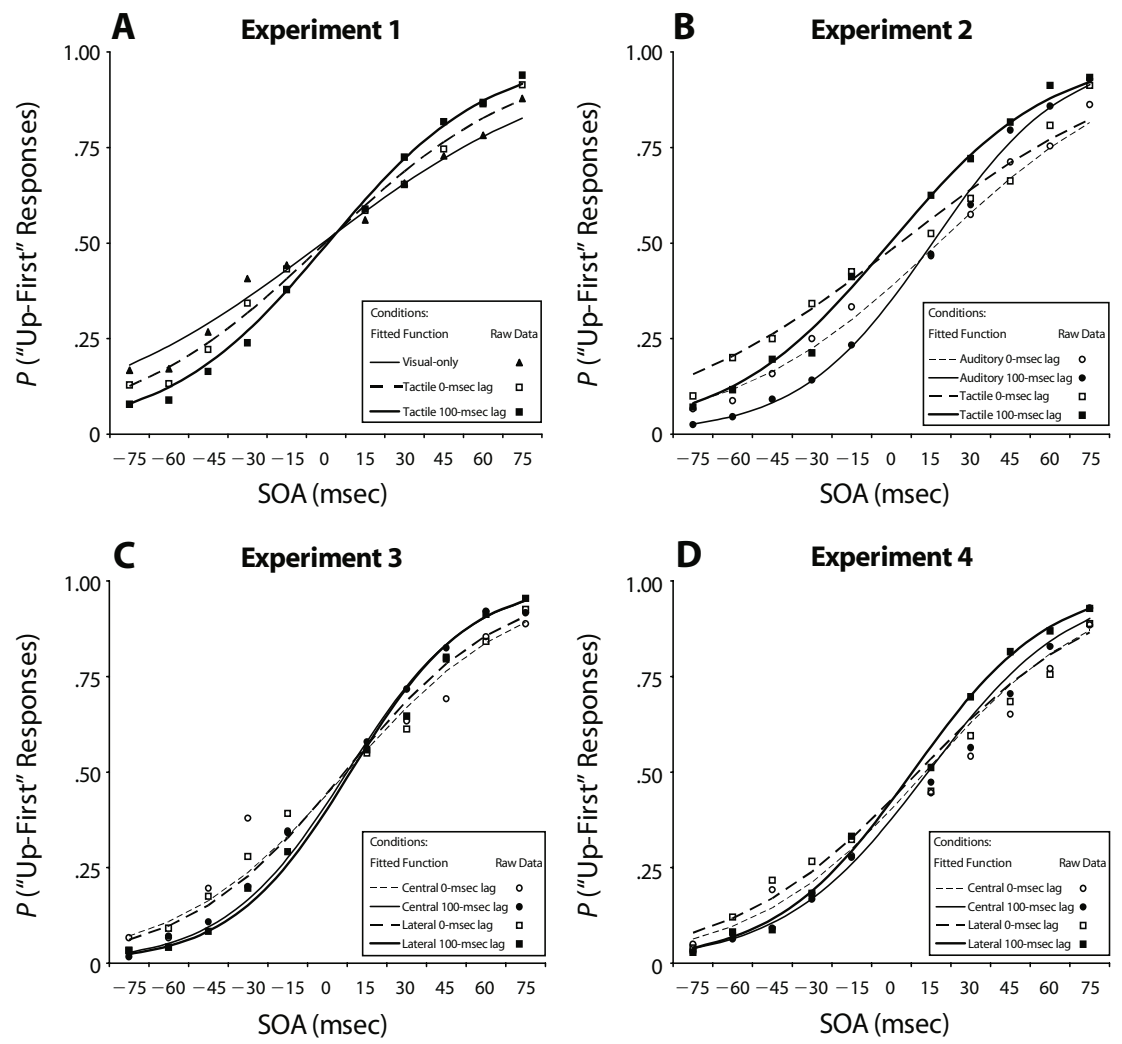

Figure 2. Averaged raw data points and fitted logistic functions. (A) Experiment 1: Tactile-visual (TV) intervals of $\sim 0$ and $\sim 100 \mathrm{msec}$, as well as a visual-only condition. (B) Experiment 2: TV intervals and auditory-visual intervals of $\sim 0$ and $\sim 100 \mathrm{msec}$. (C) Experiment 3: TV intervals at $\sim 0$ and $\sim 100 \mathrm{msec}$, with tactile stimuli delivered at a central or a lateral location. (D) Experiment 4: TV intervals at $\sim 0$ and $\sim 100 \mathrm{msec}$, with tactile stimuli delivered randomly at central and lateral locations.

indicating that there were more "down" responses for the auditory capturing stimuli than for the tactile ones (PSSs of 16.6 and $1.3 \mathrm{msec}$, respectively), a finding for which there is no clear explanation. All other effects on the PSS were nonsignificant (all $p \mathrm{~s}>.28$ ).

The ANOVA on the JNDs showed that overall sensitivity in the visual task was better with auditory capturing stimuli $(31.8 \mathrm{msec})$ than with tactile ones $(38.5 \mathrm{msec})$ $[F(1,11)=18.52, p<.001]$. This may be due to better temporal resolution of the auditory stimuli. Importantly, overall sensitivity improved by $14.7 \mathrm{msec}[F(1,11)=$ 13.08, $p<.005$ ] when the capturing stimuli (sound or touch) were presented at the $\sim 100-\mathrm{msec}$ rather than the $\sim 0$-msec interval (i.e., temporal ventriloquism). The interaction between the two factors was not significant $[F(1,11)=1.72, p=.22]$, indicating that the TV and $\mathrm{AV}$ temporal ventriloquist effects were equally sized. The TV temporal ventriloquist effect was $15.9 \mathrm{msec}(34.2 \%)$, and the AV effect was $13.4 \mathrm{msec}$ (or $34.8 \%$ ). Paired $t$ tests further showed that both effects were significantly larger than zero (both $p$ s $<.025$ ).

The results of Experiment 2 thus demonstrate that performance on the visual TOJ task became more sensitive when auditory or tactile capturing stimuli were presented at $\sim 100$ - rather than $\sim 0$-msec intervals, with no difference in the size of the improvement between the two modalities. In the following experiments, we further explored commonalities between TV and AV temporal ventriloquism by looking at whether spatial discordance between touch and light matters.

\section{EXPERIMENT 3}

In Experiments 1 and 2, tactile stimuli were delivered to the index finger, which was positioned as close as possible to the two lights. Here, we explored whether TV ventriloquism is actually hampered when the distance between the finger and the lights is increased. Tactile stimuli were presented to the index finger, which was now positioned either close to the lights (as before) or far away from them in a lateral position. If TV intersensory pairing requires spatial correspondence between the senses, one would expect no or reduced temporal ventriloquism if the touched finger is far away from the lights. Alternatively, if TV temporal ventriloquism is like AV temporal ventriloquism, spatial discordance should not then matter (Vroomen \& Keetels, 2006). 
Table 2

Mean Just Noticeable Differences (JNDs, in Milliseconds) and Standard Errors of the Means by Location of the Capturing Tactile Stimuli, for Experiments 3 and 4

\begin{tabular}{|c|c|c|c|c|c|c|}
\hline \multirow[b]{3}{*}{ Interval } & \multicolumn{3}{|c|}{ Same As Lights } & \multicolumn{3}{|c|}{ Different From Lights } \\
\hline & \multicolumn{2}{|c|}{ JND } & \multirow[b]{2}{*}{ TVE } & \multicolumn{2}{|c|}{ JND } & \multirow[b]{2}{*}{ TVE } \\
\hline & $M$ & $S E$ & & $M$ & $S E$ & \\
\hline \multicolumn{7}{|c|}{ Experiment 3} \\
\hline$\sim 0 \mathrm{msec}$ & 32.0 & 3.0 & $8.8^{*}$ & 29.9 & 2.0 & $7.4^{*}$ \\
\hline$\sim 100 \mathrm{msec}$ & 23.2 & 1.6 & & 22.5 & 1.8 & \\
\hline \multicolumn{7}{|c|}{ Experiment 4} \\
\hline$\sim 0 \mathrm{msec}$ & 32.6 & 2.7 & $5.0^{*}$ & 34.8 & 3.5 & $8.7^{*}$ \\
\hline$\sim 100 \mathrm{msec}$ & 27.5 & 2.0 & & 26.1 & 2.4 & \\
\hline
\end{tabular}

Note - The capturing tactile stimuli were presented at $\sim 0$ - or $\sim 100-\mathrm{msec}$ intervals from a central or lateral location. The temporal ventriloquist effect (TVE) is the improvement in JND between the $\sim 0$ - and $\sim 100-\mathrm{msec}$ intervals. ${ }^{*} p<.05$.

\section{Method}

Participants. Twelve students ( 9 right-handed, 3 left-handed) from the same subject pool were included in the analyses.

Stimuli and Design. The stimuli and design were as in Experiment 2, except for the following changes. Tactile stimuli were delivered to the left or right index finger, which was located either close to the fixation point (between the two lights) or in a lateral position $50 \mathrm{~cm}$ to the left (or right) of the participant's midline (see Figure 1C). No auditory capturing stimuli were used.

Procedure. The experiment consisted of three within-subjects factors: position of the stimulated finger (central or lateral), interval between the vibrotactile stimuli and the lights $(\sim 0$ or $\sim 100 \mathrm{msec})$, and SOA between the two lights (from -75 to $+75 \mathrm{msec}$, in steps of $15 \mathrm{msec}$ ). These factors yielded 40 equiprobable conditions, each presented 20 times, for a total of 800 trials. The trials were presented randomly in four blocks of 200 trials each. The location of the touched finger (central or lateral) was varied blockwise in an ABBA design, and the other factors were presented randomly. Half of the participants received the tactile stimulus on the left index finger, the other half on the right. Half of them started with a central block and the other half with a lateral block. Practice consisted of two blocks of 16 trials (one block with central tactile stimuli, the other with lateral stimuli) in which the largest SOAs $( \pm 60$ and $\pm 75 \mathrm{msec})$ were presented twice each at the $\sim 0$ - and $\sim 100$-msec intervals.

\section{Results and Discussion}

JNDs and PSSs were computed as before (see Table 2). In the ANOVA on PSSs, no effect was significant (all $F$ s $<1$ ). The ANOVA on JNDs showed that overall sensitivity improved by $8.1 \mathrm{msec}$ in the $\sim 100$-msec relative to the $\sim 0$-msec TV interval $[F(1,11)=28.70, p<.001]$. There was no overall difference between the central and lateral positions of the touched finger $[F(1,11)=1.32$, $p=.28]$, and the interaction between the two factors was not significant $(F<1)$. The temporal ventriloquist effect was $8.8 \mathrm{msec}(27.5 \%)$ when the finger was in central position, and $7.4 \mathrm{msec}(24.7 \%)$ in lateral position. Both effects were significantly larger than zero when tested separately (both $p \mathrm{~s}<.005$ ), and thus there was no sign that spatial discordance reduces TV temporal ventriloquism.

\section{EXPERIMENT 4}

Experiment 3 showed that the position of the touched finger with respect to the lights had no effect on perfor- mance in the visual TOJ task. Whether the finger was near the lights or far away from them, sensitivity on the visual TOJ task improved if the TV interval was $\sim 100 \mathrm{msec}$ rather than $\sim 0 \mathrm{msec}$. One might argue, though, that participants became adapted to the spatial discordance because the position of the finger was varied between blocks rather than between test trials. In Experiment 4, we therefore avoided adaptation by presenting vibrotactile stimuli randomly from either a central or lateral position.

\section{Method}

Participants. Fourteen students (10 right-handed, 4 left-handed) from the same subject pool were included in the analyses.

Stimuli, Design, and Procedure. The stimuli, design, and procedure were as in Experiment 3, except for the following changes. Tactile stimuli were delivered randomly to either the left or right index finger (see Figure 1D). One finger was in central position near the lights, the other was in lateral position. This resulted in 40 equiprobable conditions, each presented 20 times in random order in four blocks of 200 trials. Finger assignment (i.e., left finger in central or lateral position) alternated within each of the four blocks in an ABBA design. Half of the participants started with their left finger in central position; the other half started with their right finger there. Participants responded by pressing a foot pedal with either their toes (upper light first) or their heel (lower light first).

\section{Results and Discussion}

JNDs and PSSs were computed as before (see Table 2). A 2 (tactile stimulation at central or lateral position) $\times 2$ (TV interval of $\sim 0$ or $\sim 100 \mathrm{msec}$ ) ANOVA was performed on the JNDs and PSSs. In the ANOVA on PSSs, no effect was significant (all $p \mathrm{~s}>.05$ ). The ANOVA on JNDs showed that overall sensitivity improved by $6.9 \mathrm{msec}$ (20.5\%) in the $\sim 100$-msec relative to the $\sim 0$-msec TV interval $[F(1,13)=15.85, p<.002]$. The position of the touched finger (central or lateral) had no effect $(F<1)$, and the interaction between the two factors was not significant $[F(1,13)=2.73, p=.12]$. For the central finger position, the temporal ventriloquist effect was $5.0 \mathrm{msec}$ $(15.4 \%)$, and for the lateral position it was $8.7 \mathrm{msec}$ $(25.0 \%)$. Both effects were significantly larger than zero when tested separately (both $p \mathrm{~s}<.025$ ); if anything, temporal ventriloquism tended to be greater when the finger was far from rather than close to the light.

\section{GENERAL DISCUSSION}

Here we demonstrated, for the first time, that vibrotactile stimuli captured the apparent onset of a light. The size of this TV temporal ventriloquist effect was comparable to AV temporal ventriloquism (Aschersleben \& Bertelson, 2003; Bertelson \& Aschersleben, 2003; Fendrich \& Corballis, 2001; Morein-Zamir et al., 2003; Scheier et al., 1999; Stekelenburg \& Vroomen, 2005; Vroomen \& de Gelder, 2004; Vroomen \& Keetels, 2006), despite the various differences between sound and touch, such as their initial coding (i.e., somatotopic vs. tonotopic projections). This suggests that the capturing effect of either sound or touch is rather independent of how the information is initially coded.

The results further demonstrated that TV temporal ventriloquism was unaffected by spatial disparity between 
the visual and tactile stimuli. Temporal ventriloquism was equally large when the touched finger was close to the lights or was far away from them. This is consistent with previous findings showing that AV temporal ventriloquism is also unaffected by spatial discordance (Keetels, Stekelenburg, \& Vroomen, 2007; Keetels \& Vroomen, 2007; Vroomen \& Keetels, 2006). These results therefore lend further credit to the notion that commonality in space may not (always) be a relevant dimension for intersensory pairing to occur.

A potential concern about these findings is that our interpretation is mainly based on null effects of spatial discordance. Could it be, for example, that changing the position of the touched finger was not a powerful enough manipulation for us to measure any effect of spatial discordance? In contradiction of this idea, we tried to maximize the effect of spatial discordance in several ways. First, the touched finger was visible during the whole experiment, so the participants could not only feel, but see, its position. Second, by varying the position of the touched finger on a trial-by-trial basis, we hoped to further increase the saliency of the spatial discordance. As a result, it seems unlikely that the TV spatial disparity was not perceived. Rather, a more likely interpretation is that intersensory pairing in the temporal domain simply does not take spatial disparity into account.

The question still remains why other studies have reported effects of spatial separation on intersensory integration (see Calvert et al., 2004, and Spence \& Driver, 2004 , for reviews), whereas we have found the opposite results. We conjecture that a spatial effect on intersensory integration will be observed either if space is a dimension relevant to the task at hand (such as sound localization when distractor lights are presented concurrently at different locations; Alais \& Burr, 2004; Slutsky \& Recanzone, 2001; Stein \& Meredith, 1993; Welch \& Warren, 1986) or if spatial attention is critically involved (as in the detection of a light at an unknown location that may be cued by a sound location; Driver \& Spence, 2004; Spence \& McDonald, 2004; Spence \& Walton, 2005). Performance in these cases will depend on whether the components of the crossmodal stimulus are spatially separated or not. Notably, though, it is questionable whether intersensory integration is actually involved in the crossmodal cuing paradigm. Typically, a cue precedes a target by several hundreds of milliseconds, in which case it is unlikely that an intersensory pairing would form between the two stimuli, since the cue and target are perceived as two separate stimuli rather than a single multimodal one. Another example in which spatial separation plays a role concerns judgments of AV temporal order, which are actually improved when sounds and lights are separated (Keetels \& Vroomen, 2005; Spence, Baddeley, Zampini, James, \& Shore, 2003; Zampini, Guest, Shore, \& Spence, 2005; Zampini, Shore, \& Spence, 2003a, 2003b). In this case, the separation probably gives the participants extra spatial information on which to base their responses. Other intersensory interactions, though, such as identification tasks that do not directly involve spatial judgments, appear to be unaffected by spatial coincidence. A well-known ex- ample is the McGurk effect (i.e., information from lipreading affects speech sound identification; McGurk \& MacDonald, 1976), which occurs even when the auditory and visual speech stimuli are widely separated (Bertelson, Vroomen, Wiegeraad, \& de Gelder, 1994; Colin, Radeau, Deltenre, \& Morais, 2001; Jones \& Munhall, 1997).

To conclude, we have shown that intersensory integration in the form of TV temporal ventriloquism occurs, despite spatial discordance between the individual components of the multisensory stimulus. This demonstrates that the spatial rule about intersensory integration does not constitute a general constraint. At first sight, this might seem counterintuitive because, after all, most natural multisensory events are spatially and temporally aligned, except for some minor variations in time or space for which people can readily compensate (see, e.g., Fujisaki, Shimojo, Kashino, \& Nishida, 2004; Keetels \& Vroomen, 2008; Vroomen, Keetels, de Gelder, \& Bertelson, 2004). However, a critical assumption that underlies the idea of the spatial rule is that spatial information has the same function in vision, audition, and touch. This notion, though, is questionable. For vision, it has been argued that space is an indispensable attribute (Kubovy \& Van Valkenburg, 2001), but spatial coding in audition and touch may be different. It seems that at least sound localization has evolved to steer vision (Heffner \& Heffner, 1992), and it is also conceivable that spatial coding in touch has a role in steering vision (Stein \& Meredith, 1993). If one accepts, then, that spatial coding in the different modalities serves different functions, there is no reason why intersensory interactions would require spatial co-localization.

\section{AUTHOR NOTE}

Correspondence relating to this article may be sent to J. Vroomen, Tilburg University, Department of Psychology, Warandelaan 2, 5037 AB Tilburg, The Netherlands (e-mail: j.vroomen@uvt.nl).

\section{REFERENCES}

Alais, D., \& BurR, D. (2004). The ventriloquist effect results from nearoptimal bimodal integration. Current Biology, 14, 257-262.

Aschersleben, G., \& Bertelson, P. (2003). Temporal ventriloquism: Crossmodal interaction on the time dimension. 2: Evidence from sensorimotor synchronization. International Journal of Psychophysiology, 50, 157-163.

BEDFORD, F. L. (1989). Constraints on learning new mappings between perceptual dimensions. Journal of Experimental Psychology: Human Perception \& Performance, 15, 232-248.

Bertelson, P. (1999). Ventriloquism: A case of crossmodal perceptual grouping. In G. Aschersleben, T. Bachmann, \& J. Müsseler (Eds.), Cognitive contributions to the perception of spatial and temporal events (pp. 347-363). Amsterdam: Elsevier, North-Holland.

Bertelson, P., \& Aschersleben, G. (2003). Temporal ventriloquism: Crossmodal interaction on the time dimension. 1: Evidence from auditory-visual temporal order judgment. International Journal of Psychophysiology, 50, 147-155.

Bertelson, P., Vroomen, J., Wiegeraad, G., \& de Gelder, B. (1994, September). Exploring the relation between McGurk interference and ventriloquism. Paper presented at the Third International Congress on Spoken Language Processing, Yokohama.

Calvert, G. A., Spence, C., \& Stein, B. E. (2004). The handbook of multisensory processes. Cambridge, MA: MIT Press.

Colin, C., Radeau, M., Deltenre, P., \& Morais, J. (2001). Rules of intersensory integration in spatial scene analysis and speechreading. Psychologica Belgica, 41, 131-144. 
Driver, J., \& Spence, C. (2004). Crossmodal links in endogenous spatial attention: Evidence from human performance. In C. Spence \& J. Driver (Eds.), Crossmodal space and crossmodal attention (pp. 179-220). Oxford: Oxford University Press.

ERnST, M. O., \& BANKs, M. S. (2002). Humans integrate visual and haptic information in a statistically optimal fashion. Nature, $\mathbf{4 1 5}$, 429-433.

Fendrich, R., \& Corballis, P. M. (2001). The temporal cross-capture of audition and vision. Perception \& Psychophysics, 63, 718-725.

FInNey, D. J. (1964). Probit analysis: A statistical treatment of the sigmoid response curve (2nd ed.). Cambridge: Cambridge University Press.

Fujisaki, W., Shimojo, S., Kashino, M., \& Nishida, S. (2004). Recalibration of audiovisual simultaneity. Nature Neuroscience, 7 , 773-778.

Heffner, R. S., \& Heffner, H. E. (1992). Visual factors in sound localization in mammals. Journal of Comparative Neurology, 317, 219-232.

Hirsh, I. J., \& Sherrick, C. E., JR. (1961). Perceived order in different sense modalities. Journal of Experimental Psychology, 62, 423-432.

Jones, J. A., \& Munhall, K. G. (1997). The effects of separating auditory and visual sources on the audiovisual integration of speech. Canadian Acoustics, 25, 13-19.

Keetels, M., Stekelenburg, J., \& Vroomen, J. (2007). Auditory grouping occurs prior to intersensory pairing: Evidence from temporal ventriloquism. Experimental Brain Research, 180, 449-456.

Keetels, M., \& Vroomen, J. (2005). The role of spatial disparity and hemifields in audio-visual temporal order judgments. Experimental Brain Research, 167, 635-640.

Keetels, M., \& VRoomen, J. (2007). No effect of auditory-visual spatial disparity on temporal recalibration. Experimental Brain Research, 182, 559-565.

Keetels, M., \& Vroomen, J. (2008). Temporal recalibration to tactilevisual asynchronous stimuli. Neuroscience Letters, 430, 130-134.

Kirman, J. H. (1973). Tactile communication of speech: A review and an analysis. Psychological Bulletin, 80, 54-74.

Kubovy, M., \& VAN VAlKenburG, D. (2001). Auditory and visual objects. Cognition, 80, 97-126.

McGurK, H., \& MacDonald, J. (1976). Hearing lips and seeing voices. Nature, 264, 746-748.

Morein-Zamir, S., Soto-Faraco, S., \& Kingstone, A. (2003). Auditory capture of vision: Examining temporal ventriloquism. Cognitive Brain Research, 17, 154-163.

Murray, M. M., Michel, C. M., Grave de Peralta, R., Ortigue, S., Brunet, D., Gonzalez Andino, S., \& Schnider, A. (2004). Rapid discrimination of visual and multisensory memories revealed by electrical neuroimaging. NeuroImage, 21, 125-135.

RADEAU, M. (1994). Auditory-visual spatial interaction and modularity. Cahiers de Psychologie Cognitive, 13, 3-51.

Scheier, C. R., NiJhawan, R., \& Shimojo, S. (1999). Sound alters visual temporal resolution. Investigative Ophthalmology \& Visual Science, 40, 4169.

Slutsky, D. A., \& Recanzone, G. H. (2001). Temporal and spatial dependency of the ventriloquism effect. NeuroReport, 12, 7-10.

Spence, C., Baddeley, R., Zampini, M., James, R., \& Shore, D. I.
(2003). Multisensory temporal order judgments: When two locations are better than one. Perception \& Psychophysics, 65, 318-328.

Spence, C., \& Driver, J. (2004). Crossmodal space and crossmodal attention. Oxford: Oxford University Press.

Spence, C., \& McDonald, J. (2004). The cross-modal consequences of the exogenous spatial orienting of attention. In G. A. Calvert, C. Spence, \& B. E. Stein (Eds.), The handbook of multisensory processes (pp. 3-25). Cambridge, MA: MIT Press.

Spence, C., \& Walton, M. (2005). On the inability to ignore touch when responding to vision in the crossmodal congruency task. Acta Psychologica, 118, 47-70.

Stein, B. E., \& Meredith, M. A. (1993). The merging of the senses. Cambridge, MA: MIT Press, Bradford Books.

Stekelenburg, J. J., \& Vroomen, J. (2005). An event-related potential investigation of the time-course of temporal ventriloquism. NeuroReport, 16, 641-644.

Teder-SÄlejärvi, W. A., Di Russo, F., McDonald, J. J., \& Hillyard, S. A. (2005). Effects of spatial congruity on audio-visual multimodal integration. Journal of Cognitive Neuroscience, 17, 1396-1409.

VRoomen, J., \& DE Gelder, B. (2004). Temporal ventriloquism: Sound modulates the flash-lag effect. Journal of Experimental Psychology: Human Perception \& Performance, 30, 513-518.

Vroomen, J., \& Keetels, M. (2006). The spatial constraint in intersensory pairing: No role in temporal ventriloquism. Journal of Experimental Psychology: Human Perception \& Performance, 32, 1063-1071.

Vroomen, J., Keetels, M., de Gelder, B., \& Bertelson, P. (2004). Recalibration of temporal order perception by exposure to audiovisual asynchrony. Cognitive Brain Research, 22, 32-35.

Welch, R. B. (1999). Meaning, attention, and the "unity assumption" in the intersensory bias of spatial and temporal perceptions. In G. Aschersleben, T. Bachmann, \& J. Müsseler (Eds.), Cognitive contributions to the perception of spatial and temporal events (pp. 371-387). Amsterdam: Elsevier.

Welch, R. B., DuttonHurt, L. D., \& Warren, D. H. (1986). Contributions of audition and vision to temporal rate perception. Perception \& Psychophysics, 39, 294-300.

WeLCH, R. B., \& WARREN, D. H. (1980). Immediate perceptual response to intersensory discrepancy. Psychological Bulletin, 88, 638-667.

Welch, R. B., \& WARREN, D. H. (1986). Intersensory interactions. In K. R. Boff, L. Kaufman, \& J. P. Thomas (Eds.), Handbook of perception and human performance (Vol. 1, pp. 1-36). New York: Wiley.

Zampini, M., Guest, S., Shore, D. I., \& Spence, C. (2005). Audiovisual simultaneity judgments. Perception \& Psychophysics, 67, 531-544.

Zampini, M., Shore, D. I., \& Spence, C. (2003a). Audiovisual temporal order judgments. Experimental Brain Research, 152, 198-210.

Zampini, M., Shore, D. I., \& Spence, C. (2003b). Multisensory temporal order judgments: The role of hemispheric redundancy. International Journal of Psychophysiology, 50, 165-180.

(Manuscript received November 26, 2006; revision accepted for publication January 15, 2008.) 